\title{
The Mycobacterium marinum mel2 locus displays similarity to bacterial bioluminescence systems and plays a role in defense against reactive oxygen and nitrogen species Selvakumar Subbian, Parmod K Mehta, Suat LG Cirillo and Jeffrey D Cirillo*
}

Address: Dept. Microbial and Molecular Pathogenesis, Texas A\&M University Health Sciences Center - College of Medicine, College Station, TX 77843-1114, USA

Email: Selvakumar Subbian - SSubbian@medicine.tamhsc.edu; Parmod K Mehta - pkmehta3@hotmail.com; Suat LG Cirillo - slcirillo@medicine.tamhsc.edu; Jeffrey D Cirillo* - jdcirillo@medicine.tamhsc.edu

* Corresponding author

Published: 19 January 2007

BMC Microbiology 2007, 7:4 doi:10.1 186/|47|-2/80-7-4

This article is available from: http://www.biomedcentral.com/I47|-2/80/7/4

(C) 2007 Subbian et al; licensee BioMed Central Ltd.

This is an Open Access article distributed under the terms of the Creative Commons Attribution License (http://creativecommons.org/licenses/by/2.0), which permits unrestricted use, distribution, and reproduction in any medium, provided the original work is properly cited.
Received: 01 September 2006

Accepted: 19 January 2007

\begin{abstract}
Background: Mycobacteria have developed a number of pathways that provide partial protection against both reactive oxygen species (ROS) and reactive nitrogen species (RNS). We recently identified a locus in Mycobacterium marinum, mel2, that plays a role during infection of macrophages. The molecular mechanism of mel2 action is not well understood.

Results: To better understand the role of the $M$. marinum mel2 locus, we examined these genes for conserved motifs in silico. Striking similarities were observed between the mel2 locus and loci that encode bioluminescence in other bacterial species. Since bioluminescence systems can play a role in resistance to oxidative stress, we postulated that the mel2 locus might be important for mycobacterial resistance to ROS and RNS. We found that an M. marinum mutant in the first gene in this putative operon, melF, confers increased susceptibility to both ROS and RNS. This mutant is more susceptible to ROS and RNS together than either reactive species alone.
\end{abstract}

Conclusion: These observations support a role for the $M$. marinum mel2 locus in resistance to oxidative stress and provide additional evidence that bioluminescence systems may have evolved from oxidative defense mechanisms.

\section{Background}

Mycobacteria appear to have numerous molecular pathways responsible for their inherent resistance to reactive oxygen species (ROS) [1-3]. In most bacteria, oxidative stress induces a global regulator, OxyR, that induces detoxifying enzymes such as alkyl hydroperoxide reductase (AhpC) and catalase/hydroperoxidase I (KatG) [4,5]. During normal aerobic metabolism bacteria produce superoxide $\left(\mathrm{O}_{2} \bullet-\right)$ that is converted to hydrogen peroxide $\left(\mathrm{H}_{2} \mathrm{O}_{2}\right)$ and oxygen $\left(\mathrm{O}_{2}\right)$ by superoxide dismutase and
$\mathrm{H}_{2} \mathrm{O}_{2}$ is converted to water $\left(\mathrm{H}_{2} \mathrm{O}\right)$ and $\mathrm{O}_{2}$ by KatG [6] or AhpC [7]. The two superoxide dismutase (SOD) genes present in mycobacteria, sodA and sodC, have been suggested to play a role in resistance to ROS. A sodC mutant is more susceptible to ROS, including hydrogen peroxide $\left(\mathrm{H}_{2} \mathrm{O}_{2}\right)$, and displays a defect in growth within activated macrophages $[8,9]$. The sodA gene has been down-regulated by antisense methods, resulting in increased sensitivity to $\mathrm{H}_{2} \mathrm{O}_{2}[10]$. Mycobacteria also express a catalase, KatG, that affects resistance to ROS produced by NADPH 
oxidase activity in activated macrophages [2]. Other pathways must play an important role in resistance of $M$. tuberculosis to oxidative stress because oxyR is inactive [11], $k a t G$ is absent or mutated in numerous human clinical isolates [12-16] and $a h p C$ is expressed at very low levels $[17,18]$.

Similar to ROS, there are several pathways involved in mycobacterial resistance to reactive nitrogen species (RNS), including noxR1, noxR3 [19,20], dlaT [21], msrA $[22,23], c y s H[3]$, DNA repair, protein degradation in the proteasome and flavin cofactor synthesis [24]. In addition to its role in resistance to ROS, the mycobacterial $a h p C$ is also involved in resistance to the RNS peroxynitrite, but not nitric oxide [25]. Peroxynitrite is produced by SOD in the presence of $\mathrm{H}_{2} \mathrm{O}_{2}$ and nitric oxide, linking these two important mechanisms of oxidative stress-mediated cell death [26]. This observation may help to explain the inherent resistance of $M$. tuberculosis to peroxynitrite as compared to less pathogenic mycobacteria [27].

Bioluminescence systems can protect cells against ROS [28-32] through a catalase-like reaction between the electron donating ROS and oxidized luciferase-bound flavin mononucleotide, producing water and light [33]. The similarity of luciferases to oxidases [34] suggests that bioluminescence systems could have evolved from oxygen defense mechanisms [35]. During genetic analysis of factors that affect macrophage infection, we identified the $M$. marinum mel2 locus, which displays similarity to lux genes involved in bioluminescence [36]. In the current study, more detailed analysis of the genes in the mel2 locus suggests functional similarity between mel2 and bioluminescence systems. Based on this similarity, we asked whether the M. marinum mel2 locus is involved in resistance of mycobacteria to oxidative stress. We constructed an $M$. marinum mutant that carries a transposon insertion in the first gene in the mel2 locus, melF, by allelic exchange and demonstrated that this mutant displays increased susceptibility to both ROS and RNS. Since this mutation may have polar effects on downstream genes, we complemented this mutant with two constructs, one that carries the melF gene alone and another with the entire mel2 locus. The melF mutant defect is partially complemented by melF alone, but fully complemented by the entire mel2 locus. We recently found that the mel2 mutant displays a defect for growth in activated macrophages that is alleviated by the presence of either ROS scavengers or nitric oxide synthase inhibitors [37], suggesting that the mel2 mutant is more susceptible to ROS and RNS than wild type bacteria. The data obtained in the current study support and extend these observations through demonstration that the mel2 locus plays a role in susceptibility to several different compounds that produce ROS and RNS in laboratory media. Our results indicate that the M. mari- num mel2 locus is the first of a newly identified class of genes with similarity to bioluminescence genes involved in resistance to both ROS and RNS.

\section{Results}

\section{Similarity of the genes in the mel2 locus to} bioluminescence genes

The initial analysis of the genes present in the mel2 locus indicated that the melF, melG and $m e l H$ genes display similarity to $l u x A$ [38], luxG [39] and $l u x H$ [39] genes involved in bioluminescence [36]. In order to obtain a better understanding of these findings and explore the possibility of additional functional similarities, we conducted detailed analysis of the conserved motifs present within the melF-melK genes. We first conducted an NCBI Conserved Domain (CD) Search with MelF. We obtained a $100 \%$ alignment $\left(\mathrm{E}=1 \times 10^{-28}\right)$ for the 323 amino acid (a.a.) bacterial luciferase-like monooxygenase motif (pfam00296.11; Figure 1A). This motif is conserved in all bacterial luciferase genes, including the $l u x A$ and $l u x B$ genes from Vibrio harveyi, for which crystal structures have been previously determined $[40,41]$. Many of the residues responsible for catalytic activity and $\mathrm{FMNH}_{2}$ binding for LuxA and LuxB are also present in MelF $[42,43]$, suggesting that these proteins have related activities. Analysis of the relatedness of MelF to LuxA and LuxB places MelF on an independent branch (Figure 1B), indicating it is nearly equally related to both, with a slightly closer relationship to LuxA than LuxB.

Analysis of conserved domains within melG-melK also demonstrated striking similarity to genes involved in bioluminescence (Figure 2A). Functional domains that display similarity to LuxC (MelK), LuxD (MelH), LuxG (MelG) and LuxH (MelJ) were identified. Although there is no clear homologue of LuxE within the mel2 locus, MelH carries domains with similarity to aminopeptidases and lysophospholipases, suggesting that this protein could serve in the role of both the transferase and synthetase activities found in the lux pathway. Additional putative functional domains were present within MelG, MelH and MelI that were not present within the lux genes. Some of these differences may be due to differences in substrate specificity between these pathways and may help to explain why mycobacteria are not luminescent. With the differences and similarities between the mel 2 and lux proteins in mind, we constructed a working model for the putative biochemical roles of the proteins encoded by the mel2 locus (Figure 2B). Since mycobacteria face significant ROS during infections, we reasoned that a role in protection against ROS could help to explain the presence of conserved domains between the mel2 locus and bioluminescent systems. 


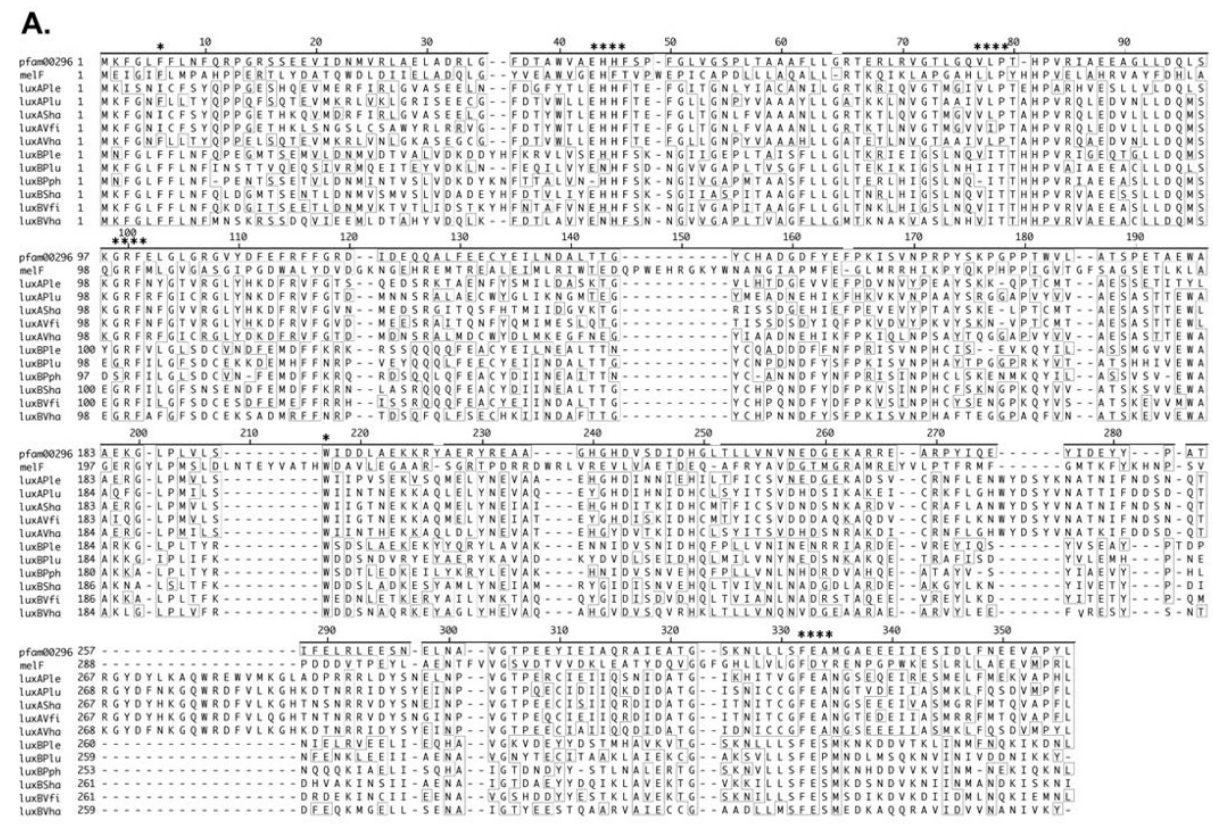

B.

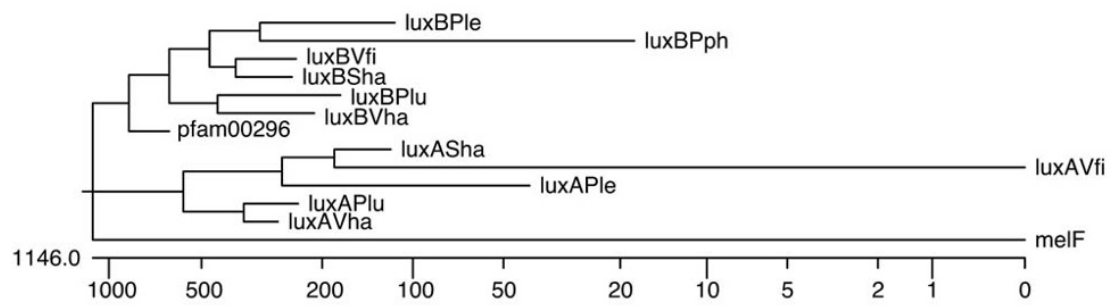

\section{Figure I}

Similarity of MelF to the bioluminescence protein luciferase. Alignment of MelF with LuxA and LuxB proteins from bioluminescent bacteria and the concensus (pfam00296) (A) and a dendogram (B) showing the relationship between them. Numbers to the left of the alignment indicate the position within each protein and above indicate the position within pfam00296. Residues that match the concensus are shown outlined by boxes. Asterixes above alignment indicate conserved regions thought to be involved in enzyme activity. Abbreviations for aligned proteins and their accession numbers are as follows: melF, M. marinum MelF (AAV32084); pfam00296, luciferase-like monooxygenases conserved domain; luxAPle, Photobacterium leiognathi LuxA (P29238); luxAPlu, Photorhabdus luminescens LuxA (AAK98554); luxASha, Shewanella hanedai LuxA (BAB40796); luxAVfi, Vibrio fishcheri LuxA (AAD48477); luxAVha, Vibrio harveyi LuxA (CAA4I597); luxBPle, Photobacterium leiognathi LuxB (P09|4I); luxBPlu, Photorhabdus luminescens LuxB (AAK98555); luxBPph, Photobacterium phosphoreium LuxB (PI 2744); luxBSha, Shewanella hanedai LuxB (BAB40797); luxBVfi, Vibrio fishcheri LuxB (AAD48478); luxBVha, Vibrio harveyi LuxB (AAA88686). The dendogram was rendered from the alignment using MegAlign (DNASTAR). Tree length is shown to the left of the log scale ruler beneath the dendogram.

\section{M. marinum mel2 mutant and complementing strains}

An M. marinum mel2 mutant was constructed by in vitro mutagenesis of the mel2 locus with mini-Mu and replacement of the wild type gene by allelic exchange [36]. We confirmed the presence of the appropriate insertion in the melF gene (Figure 2A) by Southern analysis and PCR. Since insertion mutations can have polar effects on downstream genes and the genes in the mel2 locus are very closely juxtaposed to each other, we asked whether the insertion in melF affects transcript levels for the melG-melK genes. RT-PCR with primer pairs upstream of the melF insertion mutation produces relatively similar levels of product for the M. marinum melF mutant and wild type strains, but RT-PCR with primer pairs within the downstream genes produce less product in the melF mutant than the wild type strain (Figure 3 ). These observations 
A.

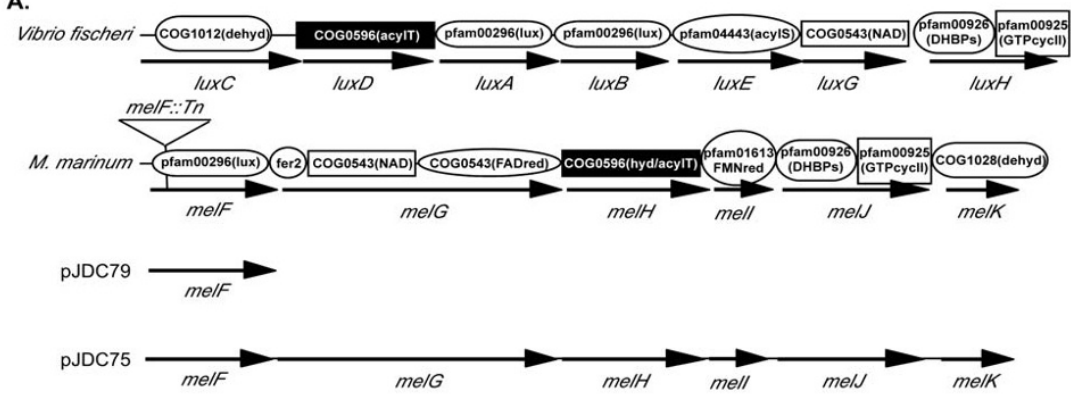

B.
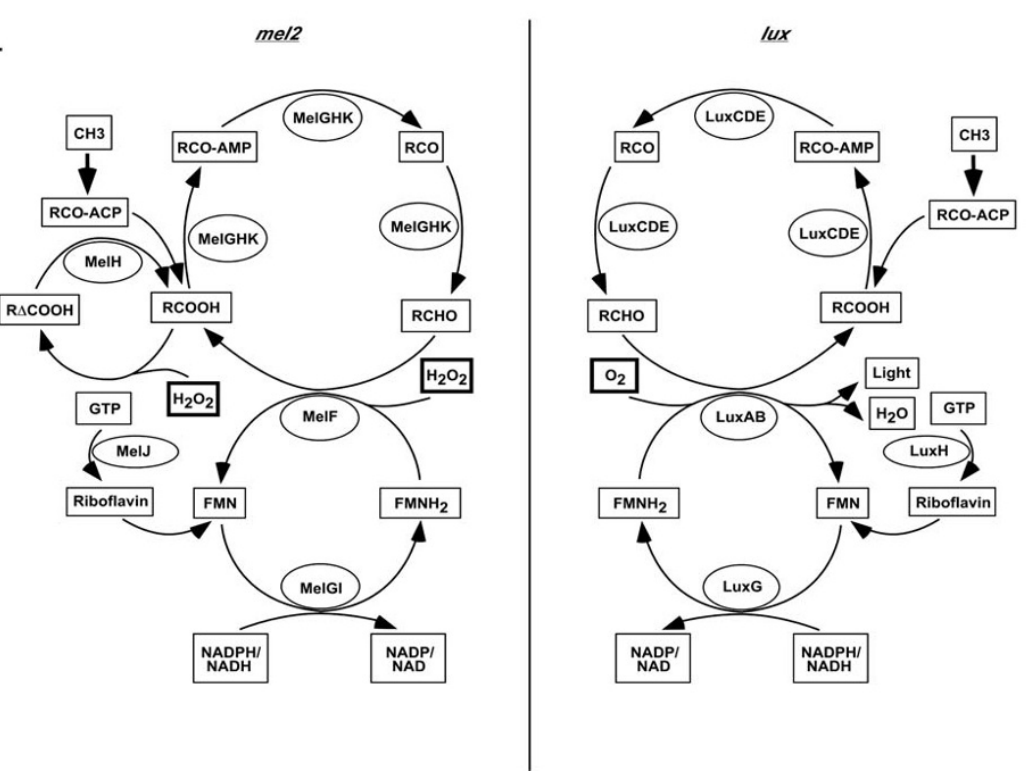

Figure 2

Conserved motifs within the mel2 locus and our working model. Conserved domains found within the Vibrio fisheri lux loci and Mycobacterium marinum mel2 locus (A) and proposed biochemical roles (B). Position of the M. marinum mel2 mutant transposon insertion is shown as a triangle above the mel2 locus (melF::Tn) and the structures of the complementing constructs, PJDC79 and pJDC75, are below the mel2 locus. The luxC-luxG region is located from the V. fisheri chromosome II and the luxH gene is from chromosome I (A). Conserved domains were identified using the NCBI conserved domain search. The designation for each conserved domain(s) is shown above each gene with an abbreviation for its proposed biochemical function in parenthesis. Abbreviations for proposed biochemical functions are as follows with additional conserved domains not shown in the figure in parenthesis: lux, luciferase; fer2, 2Fe-2S iron-sulfur cluster binding domain (cd00207); NAD, flavodoxin oxidoreductases and oxidoreductase NAD-binding domain (pfam00 I75); FADred, FAD-dependent oxidoreductases; hyd, abhydrolase alpha/beta hydrolase fold (pfam0056I); acyIT, predicted acyltransferases; FMNred, flavin reductase like domain; DHBPs, 3,4dihydroxy-2-butanone 4-phosphate synthase; GTPcycll, GTP cyclohydrolase II; dehyd, dehydrogenase; acylS, acyl-protein synthetase. We constructed a hypothetical model for how these proteins might interact to reduce reactive oxygen species (B). The resulting pathways for mel2 are similar to the lux pathways, but are better adapted to serve as a potential defense against oxidative stress through the presence of an epoxide hydrolase $(m e l H)$, in addition to the reduction of fatty acid aldehydes observed with lux. R represents a number of potential fatty acid molecules that could be used as substrates for these reactions. The LuxCDE proteins function as a complex to produce the aldehydes used to reduce oxygen by LuxAB and we have depicted a similar situation for MelGHK.

suggest that the $\mathrm{Mu}$ insertion in melF has polar effects on downstream genes and full complementation of this mutation will most likely require the entire mel2 locus. Since the $\operatorname{luxA}$ gene plays a pivotal role in biolumines- cence and similar genes, including $m e l F$, are thought to be oxidoreductases, it is possible that only the melF gene will be required for the role of mel2 in resistance to ROS. In order to differentiate between these possibilities we com- 


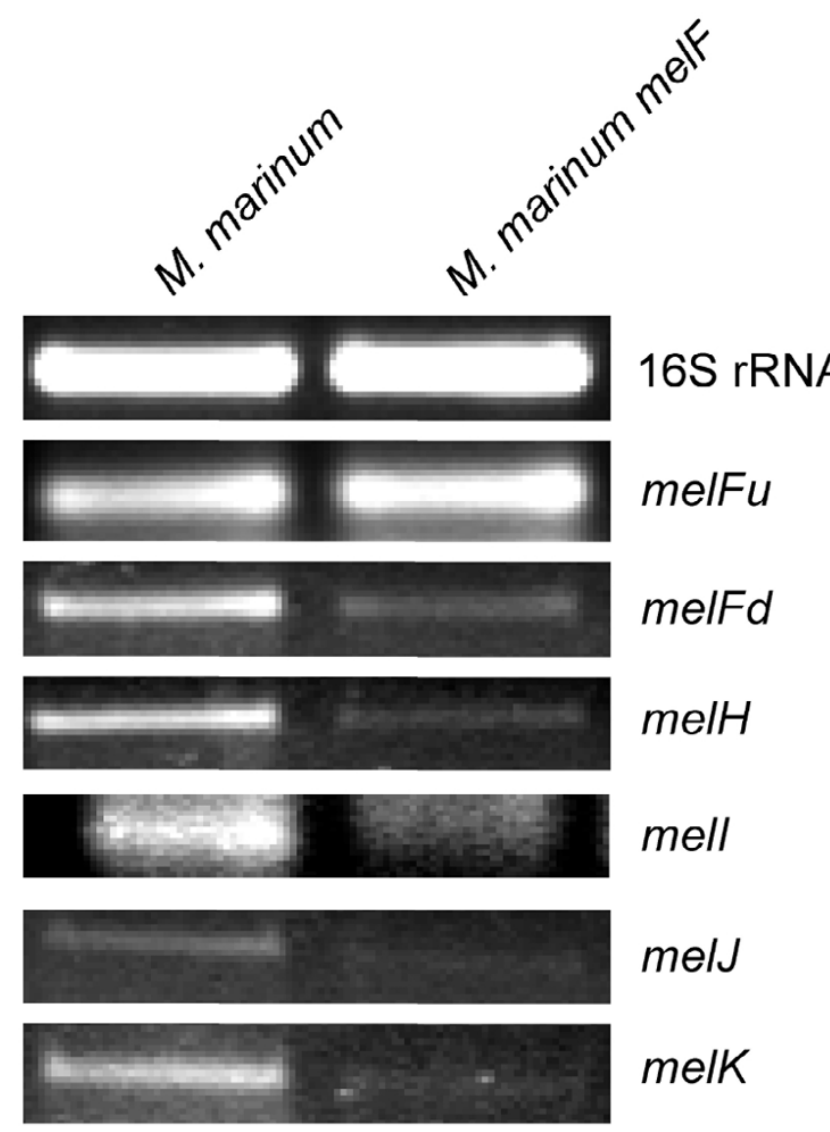

Figure 3

Examination of the polar effects due to the melF insertion mutation. RT-PCR studies to evaluate the effects of the Mu insertion on mel2 transcription upstream (melFu) and downstream of the insertion site (melFd, melH, mell, mell, melK). Equivalent amounts of RNA from $M$. marinum wild type and the $M$. marinum melF mutant were reverse transcribed and subjected to PCR with specific oligonucleotides within each gene. Equal amounts of each PCR product were loaded on $0.8 \%$ agarose gels and compared to the I6S rRNA control RT-PCR reaction for each strain (I6S rRNA) carried out on the same samples. Data shown are representative of two independent experiments.

plemented the M. marinum melF mutant with both melF alone (pJDC79) and the entire mel2 locus (pJDC75) (Figure 2).

\section{The mycobacterial mel2 locus affects susceptibility to ROS}

We first compared the ROS susceptibility of wild type $M$. marinum with that of $M$. tuberculosis and the non-pathogenic mycobacterial species $M$. smegmatis (Figure 4A). We found that M. marinum displays similar levels of resistance to $\mathrm{H}_{2} \mathrm{O}_{2}$ as $M$. tuberculosis at various concentrations and times of treatment. In contrast, $M$. smegmatis is readily killed, even at $1 \mathrm{mM} \mathrm{H}_{2} \mathrm{O}_{2}$ where the pathogenic strains are nearly completely resistant $(\mathrm{P}<0.001)$. Interestingly, we found that a mel2 mutant that carries an insertion in the melF gene is much more susceptible than wild type $M$. marinum to $\mathrm{H}_{2} \mathrm{O}_{2}$ (Figure $4 \mathrm{~B}-\mathrm{D} ; \mathrm{P}<0.01$ ). This difference is more pronounced at $5 \mathrm{mM}$ (between $76-84 \%$ for wild type vs. $4-10 \%$ survival for the mutant after $2 \mathrm{~h}$ ) than at 1 $\mathrm{mM}$ (between $85-97 \%$ for wild type vs. $52-70 \%$ survival for the mutant after $2 \mathrm{~h}$ ). Resistance to $\mathrm{H}_{2} \mathrm{O}_{2}$ cannot be restored to the mel2 mutant with the melF gene alone, even expressed from a plasmid (pJDC79), but can be restored by a single copy integrated plasmid carrying the entire mel2 locus (pJDC75). In contrast, no difference in the growth rate or survival of these mycobacterial strains in standard laboratory medium without $\mathrm{H}_{2} \mathrm{O}_{2}$ is observed (data not shown). These observations suggests that the melF gene alone is not sufficient to confer resistance to $\mathrm{H}_{2} \mathrm{O}_{2}$, and that the melF insertion mutation has polar effects on downstream genes involved in $\mathrm{H}_{2} \mathrm{O}_{2}$ resistance.

We further probed the role of the mel2 locus in resistance to ROS through the use of two additional ROS generating compounds, cumene hydroperoxide and t-BOOH. Both of these compounds are organic peroxides that produce ROS inside the bacterial cell, but are more stable in aqueous solutions than $\mathrm{H}_{2} \mathrm{O}_{2}$. Organic peroxides decompose to alkoxyl and peroxyl radicals in addition to $\mathrm{H}_{2} \mathrm{O}_{2}$ $[44,45]$. The mel2 mutant was more susceptible to both cumene hydroperoxide and $\mathrm{t}-\mathrm{BOOH}$ than wild type $M$. marinum (Figure 5; $\mathrm{P}<0.01$ ). Interestingly, at the twohour time point partial complementation of the resistance defect was observed, but once again, the entire mel2 locus confers wild type resistance levels. These observations indicate that the mel2 locus plays a role in resistance to ROS, including the diverse radicals produced by organic peroxides.

\section{The mel2 locus affects susceptibility to RNS}

Since the mel2 locus plays a role in resistance to ROS, it is also possible that it will affect resistance to RNS. The ROS and RNS pathways are linked in the reaction of nitric oxide with superoxide to produce peroxynitrite $[1,46,47]$. Because of the importance of RNS in protection against mycobacterial infections [48-51], pathways that affect susceptibility are likely to be important for pathogenesis. We examined the susceptibility of the mel2 mutant to acidified $\mathrm{NaNO}_{2}$, which is a source of nitric oxide [24,48], and SNAP, which releases nitric oxide under neutral $\mathrm{pH}$ in the presence of trace metals $[52,53]$. Similar to ROS, the mel2 mutant displays greater susceptibility than wild type $M$. marinum to RNS $(\mathrm{P}<0.01)$ and this phenotype can be complemented partially by the melF gene alone and completely by the entire mel2 locus (Figure 6). 
A.

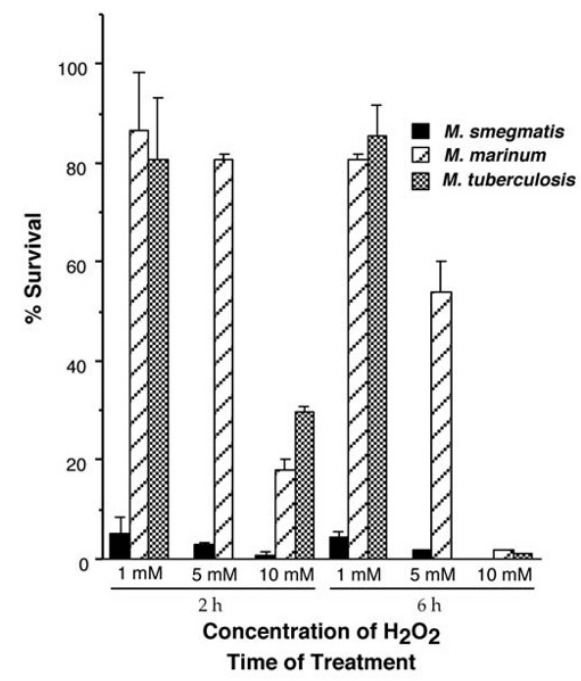

C.

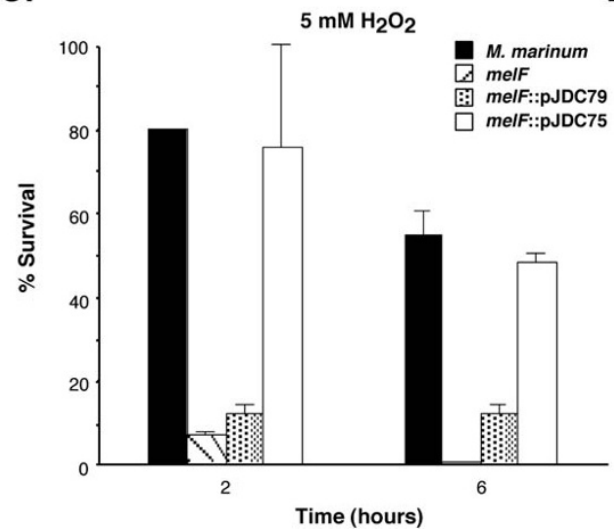

B.

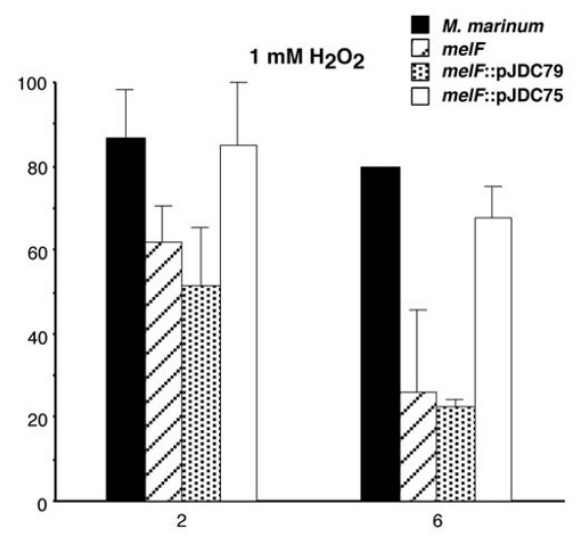

D.

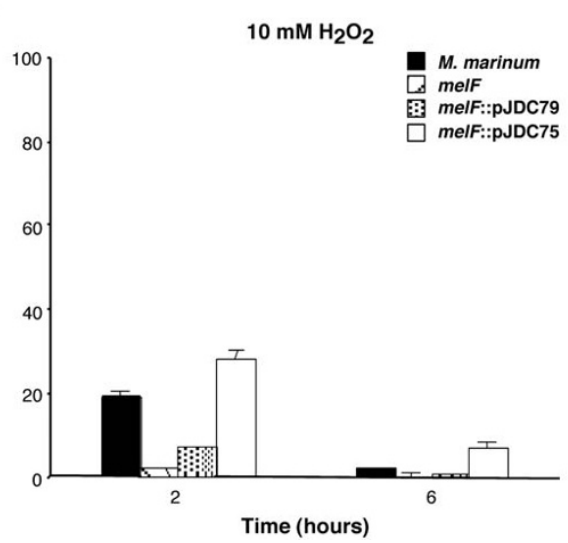

Figure 4

Susceptibility of mycobacterial species and the mel2 mutant to $\mathbf{H}_{2} \mathbf{O}_{2}$. Susceptibility of $M$. smegmatis, M. marinum and M. tuberculosis to different concentrations of $\mathrm{H}_{2} \mathrm{O}_{2}$ for 2 or $6 \mathrm{~h}(\mathrm{~A})$. The data points are absent for M. tuberculosis at $5 \mathrm{mM} \mathrm{H}_{2} \mathrm{O}_{2}$ because these bacteria were only tested at I and $10 \mathrm{mM}$ concentrations. Susceptibility of $M$. marinum wild type as compared to the mel2 mutant that carries an insertion in melF (melF), the mutant carrying a plasmid expressing melF alone (melF::pJDC79) and an integrated single copy plasmid expressing the entire mel2 locus (melF::pJDC75) to I mM (B), $5 \mathrm{mM}$ (C) and $10 \mathrm{mM}$ (D) $\mathrm{H}_{2} \mathrm{O}_{2}$. The \% survival $=($ CFU Tx/CFU initial $) \times 100$. Data are the means and standard deviations of assays done in triplicate, representative of three experiments.

The mel2 locus affects susceptibility to the combination of ROS and RNS

Since these observations suggest that the mel2 locus is involved in resistance of mycobacteria to both ROS and RNS, we examined whether the presence of both ROS and RNS simultaneously would have a more dramatic effect upon this mutant. Interestingly, the mel2 mutant is much more susceptible to treatment with both $\mathrm{H}_{2} \mathrm{O}_{2}$ and SNAP together than either compound alone (Figure 7; $\mathrm{P}<0.01$ ). These observations suggest that the mel2 mutant plays a role in susceptibility to both ROS and RNS, whether treated with them together, as most likely occurs in vivo, or separately.

\section{Discussion}

The molecular mechanisms of mycobacterial resistance to ROS and RNS have been an area of intense investigation and suggest that there are multiple pathways involved in resistance [1]. In the current study, we identified a novel set of genes in the mel2 locus that play a role in resistance to both ROS and RNS. As shown in our previous studies, this locus is also important for survival in activated macrophages and virulence in the mouse footpad model of 


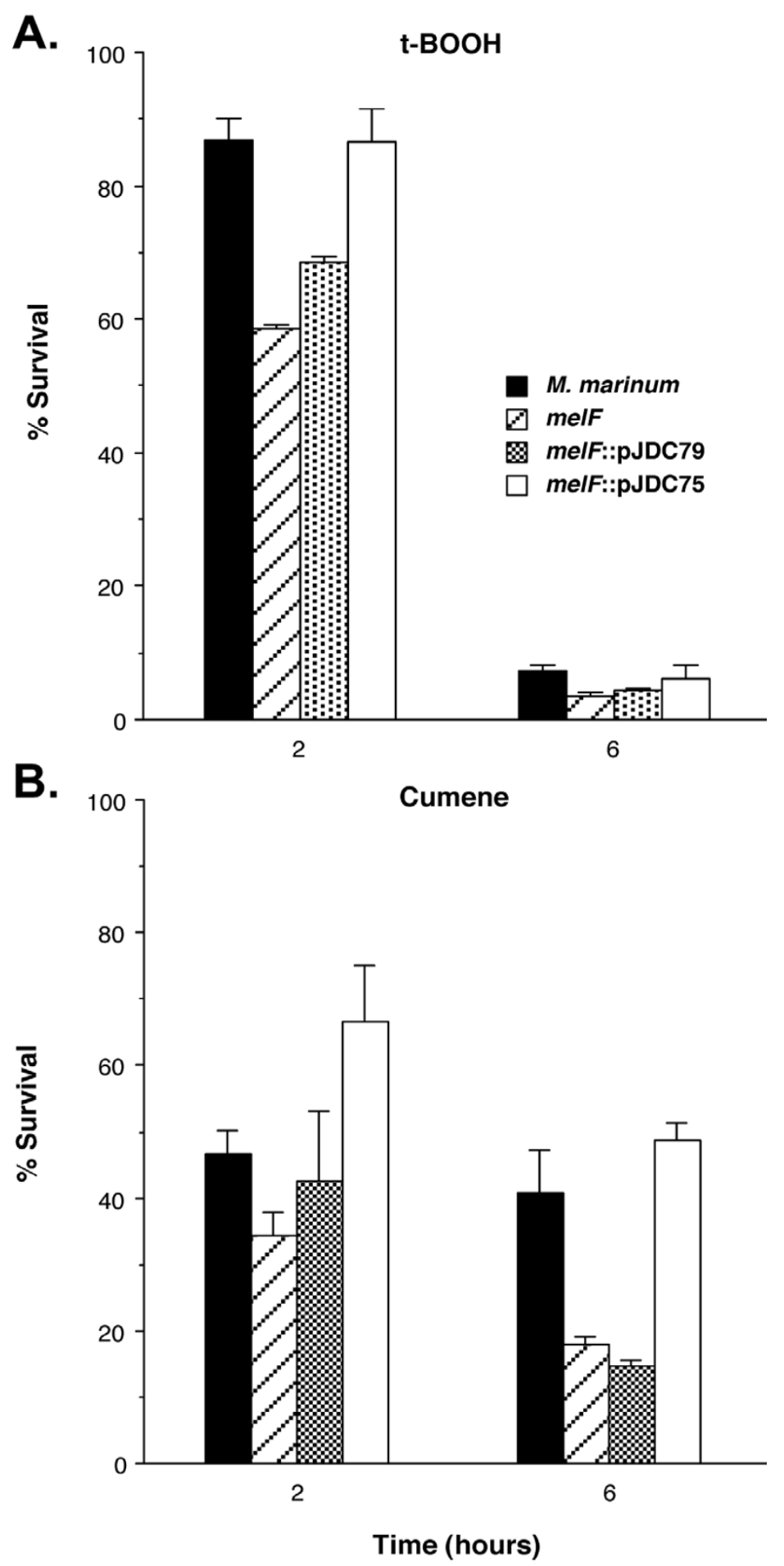

\section{Figure 5}

Susceptibility of the mel2 mutant to reactive oxygen species. Susceptibility of $M$. marinum wild type as compared to the mel2 mutant that carries an insertion in melF (melF), the mutant carrying a plasmid expressing melF alone (melF::pJDC79) and an integrated single copy plasmid expressing the entire mel2 locus (melF::pJDC75) to 10 mM tert-butyl hydroperoxide (t$\mathrm{BOOH}, \mathrm{A})$ or $100 \mathrm{mM}$ cumene hydroperoxide (Cumene, $\mathrm{B})$. The \% survival $=(\mathrm{CFU}$ Tx/CFU initial) $\times 100$. Data are the means and standard deviations of assays done in triplicate, representative of three experiments. 
A.

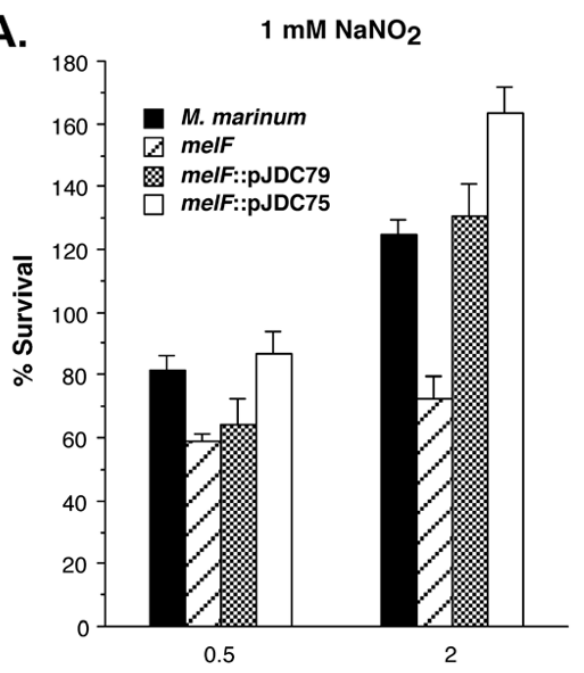

C.

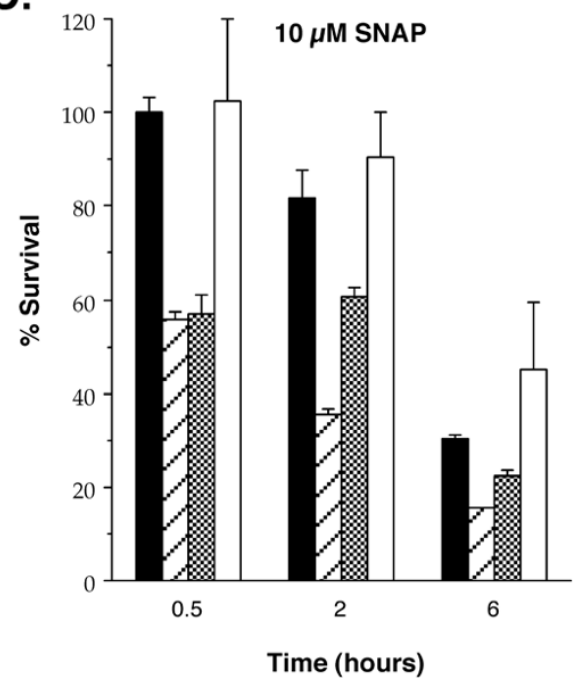

B.



D.

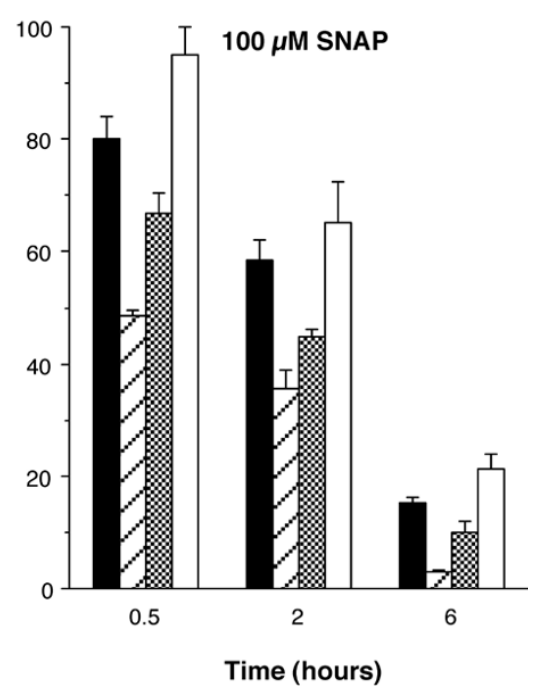

Figure 6

Susceptibility of the mel2 mutant to reactive nitrogen species. Susceptibility of M. marinum wild type as compared to the mel2 mutant that carries an insertion in melF (melF), the mutant carrying a plasmid expressing melF alone (melF::pJDC79) and an integrated single copy plasmid expressing the entire mel2 locus (melF::pJDC75) to $1 \mathrm{mM}$ (A) or $5 \mathrm{mM}$ (B) sodium nitrite $\left(\mathrm{NaNO}_{2}\right)$ and $10 \mu \mathrm{M}$ (C) or $100 \mu \mathrm{M}$ (D) S-nitroso-N-acetyl penicillamine (SNAP). The \% survival $=(\mathrm{CFU}$ Tx/CFU initial) $\times 100$. Data are the means and standard deviations of assays done in triplicate, representative of three experiments.

infection [37]. To the best of our knowledge, this is the first description of a mycobacterial pathway that impacts susceptibility to both of these reactive species. Since RNS and ROS are linked through the production of peroxynitrite from nitric oxide and superoxide $[1,46,47]$, the mel2 system may be specifically involved in resistance to this reactive species. The presence of the mel2 locus in the tuberculosis complex and M. marinum [36] and absence in avirulent mycobacteria that are more susceptible to peroxynitrite [27] supports this concept.
The similarity of the mel2 locus to bioluminescence systems at the amino acid level and the presence of conserved domains between them are intriguing observations. These data are particularly interesting in light of the recent observations that bioluminescent systems can protect cells against oxidative stress [28-32]. In search of a biological role for bioluminescence in bacteria that would explain how such an energy-consuming system could have developed evolutionarily, it has been proposed that these pathways protect against ROS generated in an aero- 


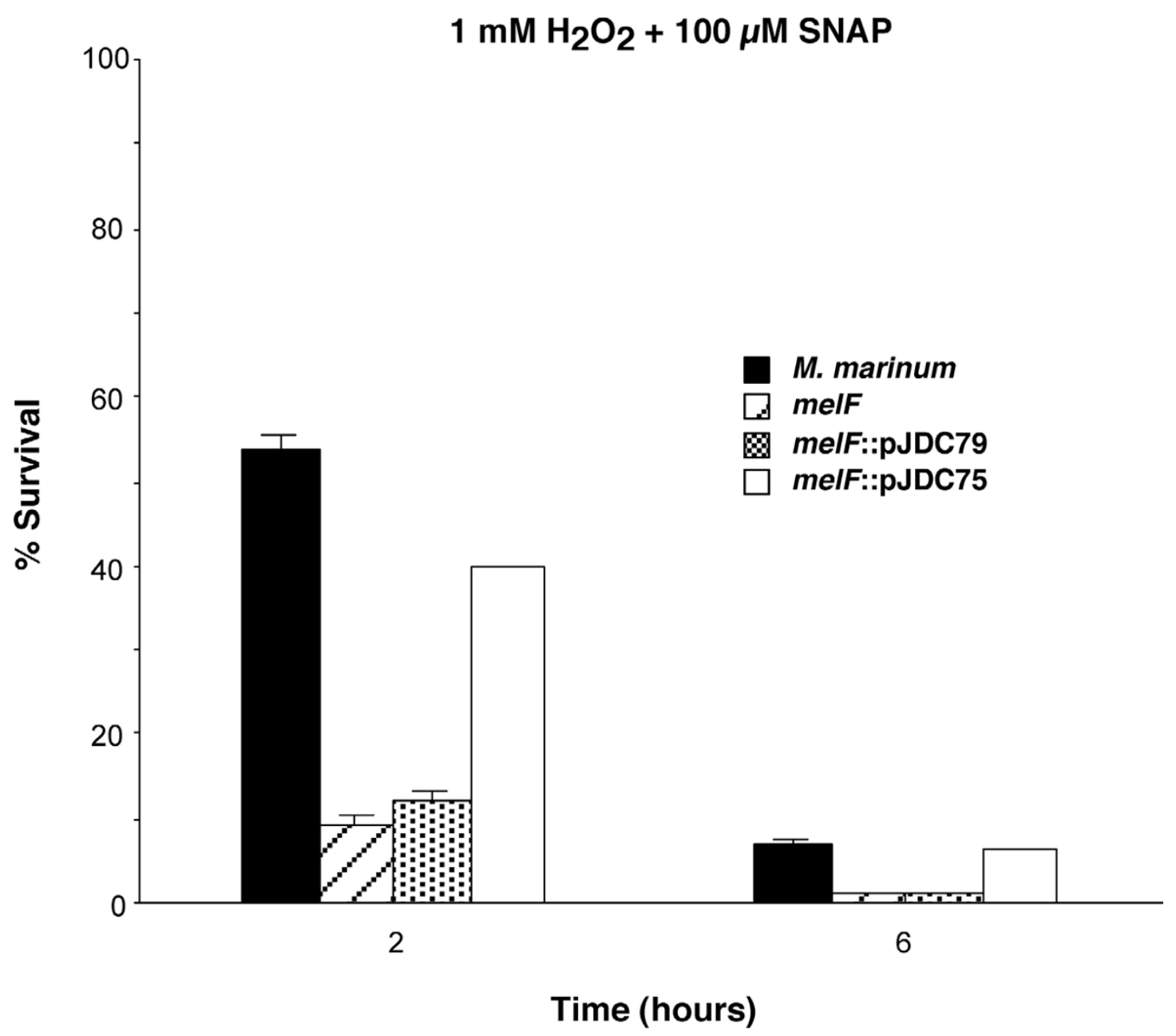

\section{Figure 7}

Susceptibility to reactive nitrogen and oxygen species together. Susceptibility of $M$. marinum wild type as compared to the mel2 mutant that carries an insertion in melF (melF), the mutant carrying a plasmid expressing melF alone (melF::pJDC79) and an integrated single copy plasmid expressing the entire mel2 locus (melF::pJDC75) to $\mathrm{H}_{2} \mathrm{O}_{2}$ plus S-nitroso-N-acetyl penicillamine $($ SNAP). The \% survival $=($ CFU Tx/CFU initial) $\times 100$. Data are the means and standard deviations of assays done in triplicate, representative of three experiments.

bic atmosphere [35,54]. Interestingly, it has been observed that ROS play a pivotal role in host-symbiont interactions with bioluminescent bacteria [55]. At present, our model for the biochemical function of mel2 (Figure 2B) is purely hypothetical and is in need of more experimental support, but the large number of conserved functional domain similarities between the lux and mel2 loci suggests that they may have related functions. However, it seems unlikely that this function is bioluminescence, since mycobacteria are not normally bioluminescent and we did not observe any bioluminescence associated with our mutant or complemented strains (data not shown). Our observation that the mel2 locus plays a role in resistance to ROS helps to explain the presence of loci similar to bioluminescence genes in nonluminescent bacterial pathogens.

The inherent resistance of $M$. marinum to ROS is impacted by a mutation in the mel2 locus. This observation suggests that mel2 has an important role in either directly scavenging oxygen radicals or repairing damage caused by them. Since the mel2 mutant affects susceptibility to $\mathrm{H}_{2} \mathrm{O}_{2}$ and the organic peroxides cumene hydroperoxide and $\mathrm{t}$ $\mathrm{BOOH}$, which generate alkoxyl radicals, peroxyl radicals and $\mathrm{H}_{2} \mathrm{O}_{2}[44,45]$, it is unclear whether mel2 is specific to a particular type of ROS. The apparent absence of specificity could be the result of this pathway utilizing an unknown oxidizable substrate that is recycled, similar to 
Table I: Oligonucleotides

\begin{tabular}{cll}
\hline Name & Target & Sequence (5' -> 3') \\
MelFuF & melFu & CAGAAGACGCGATCACGGCG \\
MelFuR & & GGGTCGGCGAACACTTCACC \\
MelFdF & melFd & CCTGCTGCCCTATCATCACC \\
MelFdR & & CCTCCAAAGCCCGAAGCCGC \\
MelFtnF & melFtn & CAGAAGACGCGATCACGGCG \\
MelFtnR & & CCTGCTGCCCTATCATCACC \\
MelGF & melG & GCGAGAAGGGCACCGCCATG \\
MelGR & & CTCAGATCACCCACGGTCAC \\
MelHF & melH & GTGACCGTGGGTGATCTGAG \\
MelHR & & GAAGGCGCAACTCACTGCCG \\
MellF & mell & CGGCAGTGAGTTGCGCCTTC \\
MellR & & CATCCAGGCTCCGTTGCGGG \\
MelJF & mell & CCCGCAACGGAGCCTGGATG \\
MelJR & & GCCGCGAGTGGCGTGTCTGC \\
MelKF & melK & GCAGACACGCCACTCGCGGC \\
MelKR & & GACGCTCACCACAGTGCGGC \\
rRNAF & rRNA & AGAGTTTGATCCTGGCTCAG \\
rRNAR & & CACGCTCACAGTTAAGCTGT
\end{tabular}

aDesignation for each oligonucleotide used in this study for RT-PCR analyses. $\mathrm{F}$ indicates a forward primer and $\mathrm{R}$ indicates a reverse primer. bTarget gene mRNA transcript for RT-PCR analyses. Oligonucleotides are listed as primer pairs. The reverse primers were used for cDNA synthesis and the forward and reverse used together for RT-PCR. The melFu target is the region of the mel2 locus transcript upstream of the transposon insertion in the melF mutant but down stream of the start codon, melFd target is the region downstream of the transposon insertion but still within melF and melFtn is across the transposon insertion in melF.

luciferin in bioluminescent systems [35], direct scavenging of $\mathrm{H}_{2} \mathrm{O}_{2}$, which all three compounds produce, or repair of damaged DNA, proteins or lipids [6]. Interestingly, luciferase can produce light using $\mathrm{H}_{2} \mathrm{O}_{2}$ alone, in the absence of luciferin, suggesting that luciferase can scavenge $\mathrm{H}_{2} \mathrm{O}_{2}$, superoxide and hydroxyl radicals [33]. Overall, these data suggest that MelF functions as a FMNdependent non-heme catalase. The presence of the mel2 locus in pathogenic mycobacteria may at least partially explain why the catalase $(k a t G)$ gene can be mutated during acquisition of isoniazid resistance [56], yet kat $G$ negative $M$. tuberculosis are responsible for numerous clinical infections in humans [12-16]. Since oxidative stress increases susceptibility of mycobacteria to isoniazid [57], it is possible that in some cases there is a relationship between isoniazid susceptibility and the mel 2 locus. This possibility can be tested by comparing the effects of a double and single katG and mel2 mutants on virulence and isoniazid resistance.

The role of bioluminescence systems from other bacteria in resistance to RNS has not been examined, but our observations with mel2 suggest that this possibility is worth investigating. Since susceptibility to both SNAP and acidified $\mathrm{NaNO}_{2}$ are impacted by the mel2 mutation, this phenotype is not the result of greater susceptibility to the acidic $\mathrm{pH}$ used with $\mathrm{NaNO}_{2}$. The fact that the mel2 mutant displays an obvious defect when exposed to a combina- tion of both ROS and RNS would imply that this locus is important for growth in environments where both of these reactive species are present, such as during infection of mammals. We found that the M. marinum luxA homologue, melF, may play an important role in resistance to both RNS and ROS, since this gene alone can partially complement what may be a polar mutation. Alternatively, this observation could be the result of low levels of expression of the remainder of genes within mel2, as a result of the polar mutation. This polar mutation would allow only low levels of the putative Mel2 protein complex to be formed and provide partial complementation once a functional melF gene is expressed. A better understanding of the biochemical roles of each of the mel2 genes and their importance in susceptibility to ROS and RNS will require analysis of each gene individually as well as in the presence or absence of each of the different Mel2 components.

\section{Conclusion}

In this study, we confirmed that the mel2 locus plays a role in the susceptibility of M. marinum to ROS and RNS. Although this locus displays similarity to bioluminescent systems in other bacterial species, further biochemical studies are necessary to demonstrate the functional significance of the conserved domains that are present. These observations suggest that mel2 represents a previously unrecognized pathway for resistance of bacterial patho- 
gens to ROS and RNS and support the concept that bioluminescence systems may have evolved from oxidative stress defense mechanisms.

\section{Methods}

\section{Strains and growth conditions}

M. marinum strain $\mathrm{M}$, a clinical isolate obtained from the skin of a patient [58], was used in these studies. M. marinum strains were grown at $33^{\circ} \mathrm{C}$ in $7 \mathrm{H} 9$ broth (Difco, Detroit, Mich.) supplemented with $0.5 \%$ glycerol, $10 \%$ albumin-dextrose complex (ADC) and $0.25 \%$ Tween 80 (M-ADC-TW) for 5 days. M. smegmatis strain $\mathrm{mc}^{2} 155$ [59] cultures were grown in M-ADC-TW for 3 days at $37^{\circ} \mathrm{C}$ and M. tuberculosis strain Erdman (ATCC35801) cultures were grown in M-ADC-TW for 10 days at $37^{\circ} \mathrm{C}$. The number of viable bacteria was determined for each assay using the LIVE/DEAD assay (Molecular Probes, Eugene, OR.) and by plating dilutions for colony forming units (cfu) on 7H9 (M-ADC) agar (Difco, Detroit, Mich.). All inocula used were $>99 \%$ viable. E. coli strains were grown in Luria-Bertani (LB, Difco) media at $37^{\circ} \mathrm{C}$. Where appropriate, kanamycin was added at a concentration of $25 \mu \mathrm{g} / \mathrm{ml}$ (E. coli) or $10 \mu \mathrm{g} / \mathrm{ml}$ (M. marinum).

\section{Construction of M. marinum mel2 mutant and complementing strains}

The M. marinum mel2 mutant carries a mini-Mu transposon insertion near the amino terminus of the melF gene as described previously [36]. Our previous studies have found no functional differences between the M. tuberculosis and M. marinum mel2 loci, both confer wild type host cell infection and growth in macrophages to the M. marinum melF insertion mutant [36,37], so either can be used for complementation studies. The $M$. marinum melF::pJDC79 strain is the melF mutant that carries the plasmid pMV262 [60] expressing the melF gene from $M$. tuberculosis that has been previously shown to complement the macrophage infection defect of the $M$. marinum mel2 mutant [36]. The M. marinum melF::pJDC75 strain is the melF mutant that carries the single-copy integrating plasmid pYUB178 [61] with the entire M. tuberculosis mel2 locus cloned into its single NheI site. Construction of all strains was confirmed by Southern analyses and PCR as described previously [36].

\section{RT-PCR analyses}

RT-PCR for the mel2 transcripts was performed using the ThermoScript RT-PCR System (Invitrogen) according to the manufacturer's instructions. Basically, 300-500 ng of DNase treated, total bacterial RNA was mixed with gene specific reverse primers, dNTP mix and 40U of RNaseOUT and incubated at $65^{\circ} \mathrm{C}$ for $5 \mathrm{~min}$ and then placed on ice prior to use. The annealed primers were extended with 15 U of Thermoscript RT at $55^{\circ} \mathrm{C}$ for 60 min followed by heat inactivation of the enzyme at $85^{\circ} \mathrm{C}$ for $5 \mathrm{~min}$. The resid- ual, non-transcribed RNA were removed with $2 \mathrm{U}$ of $E$. coli $\mathrm{RNaseH}$ at $37^{\circ} \mathrm{C}$ for $20 \mathrm{~min} .2 \mu \mathrm{l}$ of the $\mathrm{CDNA}$ was used in PCR amplification with $1 \mathrm{mM}$ appropriate forward and reverse primers and $5 U$ of Thermopol enzyme (NEB) in a total volume of $50 \mathrm{ul}$. All primers used for RT-PCR reactions are shown in Table 1. The concentrations of RNA in wild type and mutant strains were normalized against the respective 16 s rRA. 150 ng of $M$. marinum total genomic DNA of was used as positive control for the PCR reaction and RT-PCR reactions without reverse transcriptase was included in all experiments as negative control. The amplified products were analyzed by $0.8 \%$ agarose gel electrophoresis and the products measured by densitometry semiquantitatively using an Alpha Imager (Alpha Innotech) and Alpha Ease FC software.

\section{In silico analysis of the melF-melK genes}

Detailed analysis of the amino acid sequence of MelFMelK was carried out initially using protein-protein National Center for Biotechnology Information (NCBI) BLAST [62] and Conserved Domain Search [63] as described previously [36]. Once motifs of interest were identified, they were compared to the appropriate bioluminescence genes and the mel2 gene and homologues were aligned and dendograms constructed using MegAlign (DNASTAR). Domain scores were considered significant if greater than 150 and the expectation values were less than $1 \times 10^{-10}$.

\section{Susceptibility to reactive oxygen species}

Mycobacterial strains were exposed to ROS generated by $\mathrm{H}_{2} \mathrm{O}_{2}$, cumene hydroperoxide and tert-butyl hydroperoxide ( $\mathrm{t}-\mathrm{BOOH})$. The susceptibility of mycobacteria to these compounds was determined by treatment for various periods of time at the appropriate growth temperature for the mycobacterial strain used and plating dilutions on $\mathrm{M}$ ADC agar to determine CFU at each time point as compared to the original inoculum (To), i.e. percent survival $=($ CFU Tx $/$ CFU To $) \times 100$. Dimethyl sulfoxide $(\mathrm{DMSO})$ was used as a solvent for t-BOOH and was tested for effects on viability of all mycobacterial strains and no solvent affected mycobacterial viability during the time periods examined or at the final concentrations used.

\section{Susceptibility to reactive nitrogen species}

Mycobacterial strains were exposed to RNS generated by S-nitroso-N-acetyl penicillamine (SNAP) and acidification of sodium nitrite $\left(\mathrm{NaNO}_{2}\right)$ to $\mathrm{pH} 5.2$ for various periods of time. Susceptibility was determined in the same manner as that described for ROS. DMSO was used as a solvent for SNAP and had no effects on viability of mycobacteria at the concentrations and time periods used. 


\section{Statistical analyses}

All experiments were carried out in triplicate and repeated at least three times. The significance of the results was determined using the Student t-test. $P$ values of $<0.05$ were considered significant.

\section{Authors' contributions}

S.S. carried out the majority of these studies, participated in data analysis and participated in preparation of the manuscript. P.K.M. carried out some of the assays and participated in data analysis. S.L.G.C. carried out some of the assays and participated in data analysis. J.D.C. conceived the study, designed the experiments, completed the data analysis and prepared the final draft of the manuscript. All authors read the manuscript, participated in editing the manuscript and approved the final version.

\section{Acknowledgements}

This work was supported by grant Al47866 from the National Institutes of Health. We thank Drs. David McMurray and James Samuel for critical review of this manuscript.

\section{References}

I. Nathan C, Shiloh MU: Reactive oxygen and nitrogen intermediates in the relationship between mammalian hosts and microbial pathogens. Proc Natl Acad Sci U S A 2000, 97:884 I-8848.

2. $\mathrm{Ng} \mathrm{VH,} \mathrm{Cox} \mathrm{JS,} \mathrm{Sousa} \mathrm{AO,} \mathrm{MacMicking} \mathrm{JD,} \mathrm{McKinney} \mathrm{JD:} \mathrm{Role} \mathrm{of}$ KatG catalase-peroxidase in mycobacterial pathogenesis: countering the phagocyte oxidative burst. Mol Microbiol 2004, 52:1291-1302.

3. Senaratne RH, De Silva AD, Williams SJ, Mougous JD, Reader JR, Zhang T, Chan S, Sidders B, Lee DH, Chan J, Bertozzi CR, Riley LW: 5 '-Adenosinephosphosulphate reductase $(\mathrm{CysH})$ protects Mycobacterium tuberculosis against free radicals during chronic infection phase in mice. Mol Microbiol 2006, 59:1744-1753.

4. Storz G, Toledano MB: Regulation of bacterial gene expression in response to oxidative stress. Methods Enzymol 1994, 236: 196-207.

5. Christman MF, Storz G, Ames BN: OxyR, a positive regulator of hydrogen peroxide-inducible genes in Escherichia coli and Salmonella typhimurium, is homologous to a family of bacterial regulatory proteins. Proc Natl Acad Sci U S A 1989, 86:3484-3488.

6. Imlay JA: Pathways of oxidative damage. Annu Rev Microbiol 2003, 57:395-4I8.

7. Seaver LC, Imlay JA: Alkyl hydroperoxide reductase is the primary scavenger of endogenous hydrogen peroxide in Escherichia coli. J Bacteriol 200I, I 83:7173-7/8I.

8. Dussurget O, Stewart G, Neyrolles O, Pescher P, Young D, Marchal G: Role of Mycobacterium tuberculosis copper-zinc superoxide dismutase. Infect Immun 200I, 69:529-533.

9. Piddington DL, Fang FC, Laessig T, Cooper AM, Orme IM, Buchmeier NA: Cu,Zn superoxide dismutase of Mycobacterium tuberculosis contributes to survival in activated macrophages that are generating an oxidative burst. Infect Immun 200I, 69:4980-4987.

10. Edwards KM, Cynamon MH, Voladri RKR, Hager C, DeStefano MS, Tham KT, Lakey DL, Bochan MR, Kernodle DS: Iron-cofactored superoxide dismutase inhibits host responses to Mycobacterium tuberculosis. Am / Respir Crit Care Med 200I, 164:22I 3-22I9.

II. Sherman DR, Sabo PJ, Hickey MJ, Arain TM, Mahairas GG, Yuan Y, Barry CE 3rd, Stover CK: Disparate responses to oxidative stress in saprophytic and pathogenic mycobacteria. Proc Natl Acad Sci U S A 1995, 92:6625-6629.

12. Marttila HJ, Soini H, Huovinen P, Viljanen MK: katG mutations in isoniazid-resistant Mycobacterium tuberculosis isolates recovered from Finnish patients. Antimicrob Agents Chemother 1996, 40:2187-2189.

13. Marttila HJ, Soini H, Eerola E, Vyshnevskaya E, Vyshnevskiy BI, Otten TF, Vasilyef AV, Viljanen MK: A Ser3 I5Thr substitution in KatG is predominant in genetically heterogeneous multidrugresistant Mycobacterium tuberculosis isolates originating from the St. Petersburg area in Russia. Antimicrob Agents Chemother 1998, 42:2443-2445.

14. Musser JM, Kapur V, Williams DL, Krieswirth BN, van Soolingen D, van Embden JD: Characterization of the catalase-peroxidase gene (katG) and inhA locus in isoniazid-resistant and -susceptible strains of Mycobacterium tuberculosis by automated DNA sequencing: restricted array of mutations associated with drug resistance. J Infect Dis 1996, I 73:196-202.

15. Nikolayevsky V, Brown T, Balabanova Y, Ruddy M, Fedorin I, Drobniewski F: Detection of mutations associated with isoniazid and rifampin resistance in Mycobacterium tuberculosis isolate from Samara Region, Russian Federation. J Clin Microbiol 2004, 42:4498-4502.

16. Ramaswamy S, Musser JM: Molecular genetic basis of antimicrobial agent resistance in Mycobacterium tuberculosis: 1998 update. Tuber Lung Dis 1998, 79:3-29.

17. Dhandayuthapani S, Zhang Y, Mudd MH, Deretic V: Oxidative stress response and its role in sensitivity to isoniazid in mycobacteria: characterization and inducibility of ahpC by peroxides in Mycobacterium smegmatis and lack of expression in M. aurum and M. tuberculosis. J Bacteriol 1996, I 78:364|-3649.

18. Springer B, Master S, Sander P, Zahrt T, McFalone M, Song J, Papavinasasundaram KG, Colston MJ, Boettger E, Deretic V: Silencing of oxidative stress response in Mycobacterium tuberculosis: expression patterns of ahpC in virulent and avirulent strains and effect of ahpC inactivation. Infect Immun 200I, 69:5967-5973.

19. Ehrt S, Shiloh MU, Ruan J, Choi M, Gunzburg S, Nathan C, Xie Q, Riley $L$ : A novel antioxidant gene from Mycobacterium tuberculosis. J Exp Med 1997, 186:1885-1896.

20. Ruan J, St. Joh G, Ehrt S, Riley L, Nathan C: noxR3, a novel gene from Mycobacterium tuberculosis, protects Salmonella typhimurium from nitrosative and oxidative stress. Infect Immun 1999, 67:3276-3283.

21. Shi S, Ehrt S: Dihydrolipoamide acyltransferase is critical for Mycobacterium tuberculosis pathogenesis. Infect Immun 2006, 74:56-63.

22. St John G, Brot N, Ruan J, Erdjument-Bromage $H$, Tempst $P$, Weissbach $\mathrm{H}$, Nathan $\mathrm{C}$ : Peptide methionine sulfoxide reductase from Escherichia coli and Mycobacterium tuberculosis protects bacteria against oxidative damage from reactive nitrogen intermediates. Proc Natl Acad Sci U S A 200I, 98:990I-9906.

23. Douglas T, Daniel DS, Parida BK, Jagannath C, Dhandayuthapani S: Methionine sulfoxide reductase A (MsrA) deficiency affects the survival of Mycobacterium smegmatis within macrophages. J Bacteriol 2004, 186:3590-3598.

24. Darwin KH, Ehrt S, Gutierrez-Ramos JC, Weich N, Nathan CF: The proteasome of Mycobacterium tuberculosis is required for resistance to nitric oxide. Science 2003, 302:1963-1966.

25. Master SS, Springer B, Sander P, Boettger EC, Deretic V, Timmins GS: Oxidative stress response genes in Mycobacterium tuberculosis: role of ahpC in resistance to peroxynitrite and stagespecific survival in macrophages. Microbiology 2002, I 48:3 I39-3। 44

26. McBride AG, Borutaite V, Brown GC: Superoxide dismutase and hydrogen peroxide cause rapid nitric oxide breakdown, peroxynitrite production and subsequent cell death. Biochim Biophys Acta 1999, 1454:275-288.

27. Yu K, Mitchell C, Xing Y, Magliozzo RS, Bloom BR, Chan J: Toxicity of nitrogen oxides and related oxidants on mycobacteria: $M$. tuberculosis is resistant to peroxynitrite anion. Tuber Lung Dis 1999, 79:19|-198.

28. Szpilewska H, Czyz A, Wegrzyn G: Experimental evidence for the physiological role of bacterial luciferase in the protection of cells against oxidative stress. Curr Microbiol 2003, 47:379-382.

29. Barros MP, Bechara Ej: Bioluminescence as a possible auxiliary oxygen detoxifying mechanism in elaterid larvae. Free Radic Biol Med 1998, 24:767-777. 
30. Barros MP, Bechara E): Luciferase and urate may act as antioxidant defenses in larval Pyrearinus termitilluminans (Elateridae: Coleoptera) during natural development and upon 20 hydroxyecdysone treatment. Photochem Photobiol 2000, 71:648-654.

31. Barros MP, Bechara E): Daily variations of antioxidant enzyme and luciferase activities in the luminescent click-beetle Pyrearinus termitilluminans: cooperation against oxygen toxicity. Insect Biochem Mol Biol 200I, 3 I:393-400.

32. Katsev AM, Wegrzyn G, Szpilewska H: Effects of hydrogen peroxide on light emission by various strains of marine luminescent bacteria. J Basic Microbiol 2004, 44: 178-I84.

33. Watanabe $\mathrm{H}$, Nagoshi $\mathrm{T}$, Inaba $\mathrm{H}$ : Luminescence of a bacterial luciferase intermediate by reaction with $\mathrm{H} 2 \mathrm{O} 2$ : the evolutionary origin of luciferase and source of endogenous light emission. Biochim Biophys Acta 1993, I I 4 I:297-302.

34. Lei X, Cho KW, Herndon ME, Tu SC: Elicitation of an oxidase activity in bacterial luciferase by site-directed mutation of a noncatalytic residue. J Biol Chem 1990, 265:4200-4203.

35. Rees JF, De Wergifosse B, Noiset O, Dubuisson M, Janssens B, Thompson EM: The origins of marine bioluminescence: turning oxygen defence mechanisms into deep-sea communication tools. J Exp Biol | 1998, 201:|2| I-|22|.

36. El-Etr SH, Subbian S, Cirillo SL, Cirillo JD: Identification of two Mycobacterium marinum loci that affect interactions with macrophages. Infect Immun 2004, 72:6902-69/3.

37. Subbian S, Mehta PK, Cirillo SL, Bermudez LE, Cirillo JD: A Mycobacterium marinum mel2 Mutant is Defective for Growth in Macrophages Producing Reactive Oxygen and Nitrogen Species. Infect Immun 2007.

38. Escher A, O'Kane DJ, Szalay AA: The beta subunit polypeptide of Vibrio harveyi luciferase determines light emission at $\mathbf{4 2}$ degrees C. Mol Gen Genet 1991, 230:385-393.

39. Swartzman E Miyamoto C, Graham A Meighen E. Delineation of the transcriptional boundaries of the lux operon of Vibrio harveyi demonstrates the presence of two new lux genes. Biol Chem 1990, 265:35I3-35I7.

40. Fisher AJ, Thompson TB, Thoden JB, Baldwin TO, Rayment I: The I.5-A resolution crystal structure of bacterial luciferase in low salt conditions. I Biol Chem 1996, 27 I:21956-2 I968.

4I. Thoden JB, Holden HM, Fisher AJ, Sinclair JF, Wesenberg G, Baldwin TO, Rayment I: Structure of the beta 2 homodimer of bacterial luciferase from Vibrio harveyi: X-ray analysis of a kinetic protein folding trap. Protein Sci 1997, 6:13-23.

42. $\mathrm{LiCH}$, Tu SC: Probing the functionalities of alphaGlu328 and alphaAla74 of Vibrio harveyi luciferase by site-directed mutagenesis and chemical rescue. Biochemistry 2005, 44:13866-I3873.

43. $\mathrm{Li} \mathrm{CH}$, Tu SC: Active site hydrophobicity is critical to the bioluminescence activity of Vibrio harveyi luciferase. Biochemistry 2005, 44: I 2970-I2977.

44. Alia M, Ramos S, Mateos R, Bravo L, Goya L: Response of the antioxidant defense system to tert-butyl hydroperoxide and hydrogen peroxide in a human hepatoma cell line (HepG2). J Biochem Mol Toxicol 2005, 19: I 19-128.

45. Simic MG, Bergtold DS, Karam LR: Generation of oxy radicals in biosystems. Mutat Res 1989, 21 4:3-12.

46. Koppenol WH, Moreno JJ, Pryor WA, Ischiropoulos $\mathrm{H}$, Beckman JS Peroxynitrite, a cloaked oxidant formed by nitric oxide and superoxide. Chem Res Toxicol 1992, 5:834-842.

47. Ischiropoulos $\mathrm{H}$, Zhu L, Beckman JS: Peroxynitrite formation from macrophage-derived nitric oxide. Arch Biochem Biophys 1992, 298:446-45I.

48. Chan J, Xing Y, Magliozzo RS, Bloom BR: Killing of virulent Mycobacterium tuberculosis by reactive nitrogen intermediates produced by activated murine macrophages. J Exp Med 1992, 175: IIII-II22.

49. Chan J, Tanaka K, Carroll D, Flynn J, Bloom BR: Effects of nitric oxide synthase inhibitors on murine infection with Mycobacterium tuberculosis. Infect Immun 1995, 63:736-740.

50. Ehrt S, Schnappinger D, Bekiranov S, Drenkow J, Shi S, Gingeras TR, Gaasterland T, Schoolnik G, Nathan C: Reprogramming of the macrophage transcriptome in response to interferongamma and Mycobacterium tuberculosis: signaling roles of nitric oxide synthase-2 and phagocyte oxidase. J Exp Med 200I, 194:1123-1140.
5I. MacMicking JD, North RJ, LaCourse R, Mudgett JS, Shah SK, Nathan $C F$ : Identification of nitric oxide synthase as a protective locus against tuberculosis. Proc Natl Acad Sci U S A 1997, 94:5243-5248.

52. Fass U, Panickar K, Williams K, Nevels K, Personett D, McKinney M: The role of glutathione in nitric oxide donor toxicity to SN56 cholinergic neuron-like cells. Brain Res 2004, 1005:90-100.

53. Holm P, Kankaanranta H, Metsa-Ketela T, Moilanen E: Radical releasing properties of nitric oxide donors GEA 3162 , SIN-I and S-nitroso-N-acetylpenicillamine. Eur J Pharmacol 1998, 346:97-102.

54. McElroy WD, Seliger $\mathrm{HH}$ : Origin and evolution of bioluminescence. In Horizons in biochemistry Edited by: Kasha M and Pullman B. New York, Academic Press; 1962:91-101.

55. Ruby EG, McFall-Ngai MJ: Oxygen-utilizing reactions and symbiotic colonization of the squid light organ by Vibrio fischeri. Trends Microbiol 1999, 7:414-420.

56. Cohn ML, Kovitz C, Oda U, Middlebrook G: Studies on isoniazid and tubercle bacilli. II. The growth requirements, catalase activities, and pathogenic properties of isoniazid-resistant mutants. Am Rev Tuberc 1954, 70:64I-664.

57. Bulatovic VM, Wengenack NL, Uhl JR, Hall L, Roberts GD, Cockerill FR 3rd, Rusnak F: Oxidative stress increases susceptibility of Mycobacterium tuberculosis to isoniazid. Antimicrob Agents Chemother 2002, 46:2765-2771.

58. Ramakrishnan L, Falkow S: Mycobacterium marinum persists in cultured mammalian cells in a temperature-restricted fashion. Infect Immun 1994, 62:3222-3229.

59. Snapper SB, Melton RE, Mustafa S, Kieser T, Jacobs WR Jr.: Isolation and characterization of efficient plasmid transformation mutants of Mycobacterium smegmatis. Mol Microbiol 1990 , 4:1911-1919.

60. Stover CK, de la Cruz VF, Fuerst TR, Burlein JE, Benson LA, Bennet LT, Bansal GP, Young JF, Lee MH, Hatfull GF, Snapper SB, Barletta RG, Jacobs WR Jr., Bloom BR: New use of BCG for recombinant vaccines. Nature 1991, 35 I:456-460.

6I. Pascopella L, Collins FM, Martin JM, Lee MH, Hatfull GF, Stover CK, Bloom BR, Jacobs WR Jr.: Use of in vivo complementation in Mycobacterium tuberculosis to identify a genomic fragment associated with virulence. Infect Immun 1994, 62:1313-1319.

62. Altschul SF, Madden TL, Schäffer AA, Zhang J, Zhang Z, Miller W, Lipman D]: Gapped BLAST and PSI-BLAST: a new generation of protein database search programs. Nuc Acids Res 1997, 25:3389-3402.

63. Marchler-Bauer A, Anderson JB, DeWeese-Scott C, Fedorova ND, Geer LY, He S, Hurwitz DI, Jackson JD, Jacobs AR, Lanczycki CJ, Liebert CA, Liu C, Madej T, Marchler GH, Mazumder R, Nikolskaya AN, Panchenko AR, Rao BS, Shoemaker BA, Simonyan V, Song JS, Thiessen PA, Vasudevan S, Wang Y, Yamashita RA, Yin JJ, Bryant SH: CDD: a curated Entrez database of conserved domain alignments. Nucleic Acids Res 2003, 31:383-387.

Publish with Biomed Central and every scientist can read your work free of charge

"BioMed Central will be the most significant development for disseminating the results of biomedical research in our lifetime. "

Sir Paul Nurse, Cancer Research UK

Your research papers will be:

- available free of charge to the entire biomedical community

- peer reviewed and published immediately upon acceptance

- cited in PubMed and archived on PubMed Central

- yours - you keep the copyright
BioMedcentral 